\title{
Associations of Parental and Peer Rejection With Preadolescents' Loneliness: Emotional Sensitivities as Mediators
}

\author{
Samantha Ferguson' and Melanie J. Zimmer-Gembeck ${ }^{2}$ \\ 'School of Applied Psychology, Griffith University, Gold Coast, Queensland, Australia \\ ${ }^{2}$ Griffith Health Institute, Behavioural Basis of Health, Griffith University, Gold Coast, Griffith University, Gold \\ Coast, Queensland, Australia
}

\begin{abstract}
The pervasive emotional and cognitive experience of loneliness has been linked to rejection experiences and emotional sensitivity, such as rejection sensitivity (RS), defined as the tendency to anxiously expect and overreact to rejection. Moreover, RS is founded on attachment theory and has been described as a correlate of anxiety and avoidance of intimacy in close relationships. The aims of this study of 639 preadolescents (Grades 5 to 7) were to test whether the association between relationship stressors and loneliness is indirect due to the mediational role of RS, and whether the association between RS and loneliness is indirect via anxiety and avoidance of peer intimacy. As expected, regression results showed that adolescents who reported heightened perceptions of parental rejection and victimisation by peers had greater RS, which in turn was associated with adolescents' greater feelings of loneliness. Also, as predicted, adolescents with heightened RS reported more avoidance and anxiety about peer intimacy. Although anxiety about intimacy mediated the association between RS and loneliness for both boys and girls, avoidance mediated between girls', but not boys', RS and loneliness. The findings highlight the interrelations between multiple forms of emotional sensitivities, and the importance of parent-child relationships, peer victimisation, and emotional sensitivity for explaining loneliness.
\end{abstract}

Keywords: rejection sensitivity, loneliness, parent rejection, peer victimisation, anxiety, intimacy avoidance

Parents and peers are important sources of social support for most children and adolescents, but they can also be the most important sources of rejection (Berndt \& Ladd, 1989; Bowlby, 1969; Rubin, Bukowski, \& Parker, 1998). Such rejection experiences can accumulate and become one of the strongest correlates of heightened childhood loneliness, when it is defined as a sad or aching sense of disassociation from others, as well as a longing for contact or closeness with others (Asher \& Paquette, 2003; Marston, Hare, \& Allen, 2010; Sentse, Lindenburg, Omvlee, Ormel, \& Veenstra, 2010). In one prominent theory, derived in part from Bowlby's (1969) attachment theory, Cassidy and Berlin (1999) proposed a model to explain when and why parent and peer rejection culminate in loneliness and maladjustment across the lifespan. In this model, young children's experiences of caregiving, particularly parental insensitivity and rejection, are proposed as the foundations for the development of cognitive representations of relationships with oth- ers. These cognitive representations of others as insensitive and rejecting make it more likely that peers will be perceived as rejecting (or that actual peer rejection may occur), and these adverse social experiences with parents and peers will yield the emergence of loneliness via the intervening role of cognitive and emotional sensitivities to interpersonal and other forms of stress.

Despite this theory and existing research on social adversity and loneliness among children and adolescents (e.g., Hawker \& Boulton, 2000; Heinrich \& Gullone, 2006; Laursen \& Hartl, 2013), there has been relatively little study of multiple emotional sensitivities that can explain how social adversity, in the form of rejection,

ADDRESS FOR CORRESPONDENCE: Ms Samantha Ferguson, School of Applied Psychology, Griffith University, Gold Coast Campus, Parklands Drive, Southport QLD 4222, Australia. Email: samantha.ferguson@griffithuni.edu.au 
yields greater loneliness among preadolescents. In addition, very little research has simultaneously considered multiple relationships, including experiences with parent and peers. Thus, the general purpose of this study was to investigate social influences on loneliness and to determine how emotional sensitivities, including rejection sensitivity, anxiety about intimacy and avoidance of intimacy, may play a role in the associations between parent and peer rejection experiences, dyadic friendship qualities, and loneliness.

\section{Emotional Sensitivity}

Theories have identified cognitive biases, emotional sensitivity or cognitive-emotional processing patterns as important to consider in order to better account for the association between rejecting experiences with parents and peers and loneliness. For example, rejection sensitivity (RS) theory identifies RS, defined as the tendency to anxiously or angrily expect, readily perceive, and overreact to rejection, as one socioemotional-cognitive processing pattern that could more fully explain how loneliness occurs from rejection experiences (Downey \& Feldman, 1996; London, Downey, Bonica, \& Paltin, 2007).

\section{Rejection sensitivity.}

Derived in part from Bowlby's (1969) attachment theory, RS is viewed as the internalisation of early parental and peer rejection experiences (Downey \& Feldman, 1996). In addition to drawing from attachment theory, the RS model also draws from social cognitive theory (Downey, Bonica, \& Rincón, 1999) to propose that early parental and peer rejection experiences affect current and future social information processing through (1) the attributions that people make for others' behaviours, and (2) the formation and subsequent activation of relational schemas.

Research has distinguished between two affective responses that indicate greater RS (Downey, Lebolt, Rincón, \& Freitas, 1998). That is, some rejection sensitive individuals may respond to implied rejection with anger and others with anxiety, which in turn are associated with different social consequences (Downey et al., 1999; London et al., 2007). Both anxious and angry forms of RS have been associated with internalising and externalising problems (Harper, Dickson, \& Welsh, 2006; London et al., 2007). However, anxious RS has been more strongly associated with internalising difficulties (i.e., 'flight' responses, such as social anxiety) and angry RS with externalising problems (i.e., 'fight' responses, such as aggression; London et al., 2007; McDonald, Bowker, Rubin, Laursen, \& Duchene, 2010; ZimmerGembeck \& Nesdale, 2013).

Despite the identification of these two forms of RS and their differential associations with maladjustment, there has been only one previous study of anxious and angry RS and their associations with adolescents' peer rejection and loneliness (London et al., 2007), and no published study of parental rejection, anxious RS, angry RS, and loneliness. London and colleagues (2007) found that peer rejection experiences increased angry RS, but only for boys, and also, that anxious and angry expectations of rejection predicted more loneliness. Thus, RS was identified as a potential mediator of the rejection-loneliness link, which was tested and refined in the present study.

\section{Avoidance of intimacy and anxiety about intimacy.}

The RS model describes multiple steps in a pathway from rejection experiences to socioemotional adjustment, which highlights RS as a conduit that helps to shape responses and ways of thinking about others and, eventually, affects adjustment. This complicated pathway of social experience, emotion and cognition involves the development of ways of thinking about rejection specifically, but also can have implications for ways of thinking about intimacy more generally. Thus, RS seems to facilitate emotions and behaviours that mark insecurity about relationships, which manifest in the tendency to either avoid or anxiously maintain relationships (Downey et al., 1999).

Brennan, Clark, and Shaver (1998) originally established these two orthogonal dimensions of insecure adult attachment, which were subsequently applied to an adolescent sample with a focus on romantic relationships (Downey et al., 1999). Both avoidance of intimate relationships and anxiety about intimate relationships can be problematic for maintaining current and developing future high quality relationships. For example, avoidance may lead to missed opportunities in gaining acceptance, establishing intimacy, and learning competencies such as conflict resolution. Alternatively, anxiously seeking to maintain a relationship at a time when one is not developmentally mature enough may result in dependency on the relationship for emotional wellbeing, as well as coercive or overly compliant behaviour (Downey et al., 1999).

The RS model supports hypotheses of mediational links between rejection experiences and avoidance and anxiety via RS. No previous study has examined avoidance and anxiety as emotional sensitivities that might identify more loneliness among some preadolescents. In the present study, we tested whether RS was associated with avoidance and anxiety and whether anxious and angry RS and intimacy avoidance and anxiety have the potential to augment feelings of loneliness.

\section{Gender Differences and Moderation}

As previously identified, loneliness and emotional sensitivity are expected to be elevated among children who have experienced parent rejection and/or peer relationship problems. However, gender-specific trends become apparent in the mechanisms through which emotional sensitivity is expressed. In previous research, females high in RS have reported higher rates of depressive symptoms but not aggression, whereas high RS males report more 
aggression but not depression (Downey et al., 1999). In this connection, we conducted analyses for boys separate from girls to allow for the possibility of gender differences in associations. In particular, we expected differences to be found for avoidance of peer intimacy. In the study of intimacy avoidance and anxiety, gender differences have been more frequently suggested and found when examining intimacy avoidance. Rubin and Coplan (2004) reported that rejected males are more likely to avoid social interaction than rejected females. Many researchers attribute these findings to socialisation, emphasising the greater importance of autonomy for boys and the greater importance placed on intimate relationships for girls (Johnson, LaVoie, Spenceri, \& Mahoney-Wernli, 2001; Ozen, Sumer, \& Demir, 2010). Rose and Rudolph (2006) suggested that girls value intimacy and supportiveness in their dyadic relationships more so than boys, and reported higher rates of loneliness when avoiding interpersonal relationships. Furthermore, avoidance of intimacy may act as a protective mechanism for boys in two ways: first, by potentially limiting activation of RS expectations; and second, by maintaining consistency with male gender norms of self-reliance and autonomy (Duarte \& Thompson, 1999; Ludlow \& Mahalik, 2001; Rosenfeld, 1979; Ward, Bergner, \& Kahn, 2003). Taken together, these findings suggest that the association between RS and loneliness may be mediated by intimacy avoidance only among girls.

Furthermore, some studies have claimed that girls are more likely than boys to anxiously maintain personal relationships (Kingery, Erdley, \& Marshall, 2011). This may be because for girls, personal validation is more likely to be premised by success or failure in social relationships (Gilligan, 1982; Purdie \& Downey, 2000). However, detrimental correlates of anxiety-based interpersonal relationships have been found for both genders, as both males and females who anxiously maintain relationships report more loneliness (Downey, Feldman, \& Ayduk, 2000). Thus, we do not expect gender differences in the relationship between RS and anxiety or the relationship between anxiety and loneliness.

\section{Summary of the Study Aims and Hypotheses}

In summary, we had three general aims and seven hypotheses in the current study. Placing the focus on the social and social-cognitive consequences of rejection experiences, the first aim was to examine associations between rejection experiences and RS, as proposed in the RS model (Downey et al., 1999). It was hypothesised that both parental rejection behaviours and peer victimisation would be positively associated with loneliness (Hypothesis 1). Also, it was predicted that RS (anxious and angry) would be positively associated with loneliness (Hypothesis 2) and mediate the association between rejection experiences (parent and peer) and loneliness (Hypothesis 3).
The second aim of the current study was to test whether children higher in RS were also more anxious about their peer relationships. The RS model depicts the use of anxious strategies for current relationships as a consequence of RS (Downey et al., 1999). This negative social consequence of the RS model has never been tested in children or adolescents. Yet, one previous study of university students (Zimmer-Gembeck \& Wright, 2007) did examine associations of RS with anxiety about relationship intimacy. Based on these findings, it was anticipated that heightened RS would be associated with greater anxiety about relationships (Hypothesis 4). It was also anticipated that, for both boys and girls, anxiety would mediate the association between RS (anxious and angry) and loneliness (Hypothesis 5).

The third study aim incorporated avoidance of intimacy and gender differences. Both boys and girls higher in RS (anxious and angry) were expected to avoid intimacy in peer relationships, as was found in a previous study of university students (Zimmer-Gembeck \& Wright, 2007; Hypothesis 6). Yet, girls have been found to place more emphasis on intimate relationships and to experience more detrimental outcomes if intimacy is avoided (Johnson et al., 2001). Thus, it was also anticipated that intimacy avoidance would mediate the association between RS and loneliness for girls but not for boys (Hypothesis 7).

\section{Method}

\section{Participants}

The participants were 639 children from three schools (one public and two private) in an urban area of Australia. Children's ages ranged from 9 to 13 years ( $M=10.8$ years, $S D=1$ year), with 329 boys $(51.5 \%)$ and 310 girls (48.5\%). Participants were attending Grade $5(n=231)$, Grade $6(n=209)$, and Grade $7(n=$ 199). According to school-level demographic information, students attending the school ranged from low to middle-high socioeconomic status. Of the sample, 90\% were white Australian and 10\% were Aboriginal Australian, Maori or Pacific Islander, Asian or from other sociocultural backgrounds.

\section{Measures}

\section{Loneliness.}

Loneliness was measured with the Loneliness and Social Dissatisfaction Questionnaire (LSDQ; 13 items; Cassidy \& Asher, 1992). Responses ranged from 1 (not at all true) to 5 (very true). An example item was 'It is hard to get kids to like me'. A total score was formed by reversing items where needed and averaging responses, with higher scores indicating more loneliness. Cronbach's $\alpha$ in the present study was .90 . 


\section{Rejection Sensitivity (RS).}

Anxious and angry RS was measured with the Children's Rejection Sensitivity Questionnaire (CRSQ; Downey et al., 1998). Given the reduced time available in the classroom, six of the original twelve vignettes portraying the possibility of rejection by teachers (three vignettes) or peers (three vignettes) were included in the current study. After each vignette, questions were asked regarding the expected likelihood of rejection, and the degree of anxiety and anger over its possible occurrence. An example reads: 'Imagine that you're in class. Your teacher asks for a volunteer to help plan a party for your class. Lots of kids raise their hands so you wonder if the teacher will choose YOU.' Following each vignette, children respond to three questions designed to measure their affect and rejection expectation. The first two questions assess anxious and angry responses by asking how nervous and how mad they would feel in this situation. Responses to these items ranged from 1 (not at all) to 5 (yes/extremely). The third question is used to assess the perceived likelihood of rejection in the situation. Responses ranged from 1 (NO!) to 5 (YES!). As suggested by the authors of this measure (Downey \& Feldman, 1996), items were scored by weighting children's expectations of rejection by their responses of worry or anger. Two scores for each vignette are calculated by reversing the response to the expectation item before multiplying this score by the children's responses regarding anxiety and anger. The six weighted items were then averaged to produce anxious and angry RS scores for each child. Higher scores represent more RS. In the current study Cronbach's $\alpha$ was .75 for RS anxious and was .77 for RS angry.

\section{Parent rejection.}

Parental rejection was measured with eight items from the Parents as Social Context Questionnaire (Skinner, Johnson, \& Snyder, 2005). Four items assessed excessive disapproval or criticism and four items assessed warmth. An example item was 'Sometimes I wonder if my parents like me'. All items had response options that ranged from 1 (not at all true) to 5 (very true). Items from the warmth subscale were reversed and all items were then averaged to form a total score with higher scores indicating more rejection. Cronbach's $\alpha$ was .80.

\section{Peer rejection experience.}

Peer rejection experience was measured with the Aggression and Victimisation Scale (Orpinas \& Horne, 2006). Thus, this measure is referred to as peer victimisation in the following sections. Three items assessed the frequency of overt aggression (i.e., physical or verbal force with the potential to cause harm). An example item was 'Kids threaten to or do push, shove or hit me'. Also, four items assessed relational forms of victimisation (i.e., harm through hurting peer relationships; Crick \& Grotpeter, 1996). An example item was 'Kids leave me

\section{TABLE I}

Descriptive Statistics for Boys $(n=329)$ and Girls $(n=310)$

\begin{tabular}{lccccc}
\hline & \multicolumn{2}{c}{ Boys } & & \multicolumn{2}{c}{ Girls } \\
\cline { 2 - 3 } \cline { 6 - 6 } Variable & $M$ & $S D$ & & $M$ & $S D$ \\
\hline Loneliness & 2.08 & 0.79 & & 1.99 & 0.74 \\
Parent rejection & 1.63 & 0.68 & & 1.50 & 0.59 \\
Peer victimisation & 1.71 & 0.73 & & 1.73 & 0.77 \\
RS anxious & 6.15 & 2.67 & & 6.54 & 2.60 \\
RS angry & 5.47 & 2.44 & & 5.39 & 2.31 \\
Avoidance & 1.60 & 0.73 & & 1.48 & 0.65 \\
Anxiety & 1.90 & 0.78 & & 2.14 & 0.83 \\
& & & &
\end{tabular}

out on purpose'. Responses ranged from 1 (not at all) to 5 (a lot). Items were averaged to create a single peer victimisation score with higher scores indicating more peer victimisation. Cronbach's $\alpha$ was .86.

\section{Intimacy avoidance and anxiety.}

Seven items from the Experiences in Close Relationships Inventory (ECRI; Brennan et al., 1998) were modified to measure intimacy avoidance and anxiety in relation to peer relationships and friendships (rather than in dating and romantic relationships). Intimacy avoidance (three items) referred to discomfort with being close to or dependent on peers. An example reads: 'If another kid tries to be my close friend, I try to avoid him or her.' Anxiety (four items) referred to being dependent or over-compliant due to fear of rejection or abandonment, in respect to relationships with others. An example reads: 'I often want my friends to tell me they like me.' Response options ranged from 1 (not at all true) to 5 (very true). Items were averaged to produce summary scores for intimacy avoidance and anxiety. Higher scores indicated higher intimacy avoidance or higher anxiety. For the current study Cronbach's $\alpha$ was .51 for intimacy avoidance and .67 for anxiety.

\section{Procedure}

Following ethical approvals, and parental and child consent, children completed questionnaires during class time. Instructions and questions were read aloud by members of the research team, consisting of Project Investigators, honours students, and undergraduate volunteers who assisted in the collection of the data. Children were informed that they could talk to project staff following study participation, if they so requested. Completion of the items included in the present study took approximately 30 minutes.

\section{Overview of Analyses}

The distributions of all of the primary measures had significant skew. Log transformations were applied that reduced the skew, and these transformed variables were used in all analyses except for the reporting of the descriptive statistics (see Table 1). Prior to testing the seven 


\section{TABLE 2}

Pearson's Correlations Between all Variables for Boys $(n=329)$ and Girls $(n=310)$

\begin{tabular}{llllllll}
\hline Variable & $\mathrm{I}$ & 2 & 3 & 4 & 5 & 6 & 7 \\
\hline I. Loneliness & $\mathrm{I}$ & $.35^{* *}$ & $.47^{* *}$ & $.44^{* *}$ & $.31^{* *}$ & $.16^{* *}$ & $.28^{* *}$ \\
2. Parent rejection & $.37^{* *}$ & $\mathrm{I}$ & $.23^{* *}$ & $.25^{* *}$ & $.23^{* *}$ & $.15^{* *}$ & $.15^{* *}$ \\
3. Peer victimisation & $.46^{* *}$ & $.31^{* *}$ & $\mathrm{I}$ & $.36^{* *}$ & $.33^{* *}$ & $.18^{* *}$ & $.38^{* *}$ \\
4. RS anxious & $.41^{* *}$ & $.33^{* *}$ & $.30^{* *}$ & $\mathrm{I}$ & $.63^{* *}$ & $.17^{* *}$ & $.40^{* *}$ \\
5. RS angry & $.30^{* *}$ & $.22^{* *}$ & $.26^{* *}$ & $.65^{* *}$ & $\mathrm{I}$ & $.18^{* *}$ & $.36^{* *}$ \\
6. Avoidance of peer intimacy & $.31^{* *}$ & $.23^{* *}$ & $.19^{* *}$ & $.26^{* *}$ & $.20^{* *}$ & $\mathrm{I}$ & $.29^{* *}$ \\
7. Anxiety about peer intimacy & $.31^{* *}$ & $.26^{* *}$ & $.36^{* *}$ & $.37^{* *}$ & $.34^{* *}$ & $.29^{* *}$ & $\mathrm{I}$ \\
\hline
\end{tabular}

Note: Correlations for boys are presented above the diagonal and correlations for girls are presented below the diagonal. ${ }^{*} p<.05 .{ }^{* *} p<.01$.

hypotheses, $t$ tests were estimated to compare boys and girls on all measures.

Pearson's $r$ correlations and hierarchical regression models, derived using the SPSS PROCESS macro (Hayes, 2012), were used to test all hypotheses. PROCESS is an SPSS macro that completes regression analyses to estimate the magnitude and significance of the $a$ (independent variable [IV] to mediator), $b$ (mediator to dependent variable [DV]), $c$ (IV to DV), and c' (IV to DV, controlling for mediators) associations. Also, indirect effects $(a b)$ and 95\% bias-corrected confidence intervals were estimated using bootstrapping available in PROCESS. Bootstrapping is a resampling method that simulates repetition of the original sample, making no assumptions about the shape of the sampling distribution. As recommended by Preacher, Rucker, and Hayes (2007), 5,000 bootstrap samples were used in the present study. All models were estimated for the full sample and for boys and girls separately. All models were estimated for the full sample and for boys and girls separately. When results were similar for boys and girls (i.e., both statistically significant with comparable standardised regression weights), only the full sample results (i.e., boys and girls combined) are presented. When these criteria were not met, results from separate analyses (i.e., boys and girls separately) were presented.

\section{Results}

\section{Gender Differences}

Girls, relative to boys, reported more anxious RS, $t(637)=2.81, p<.001$, and more anxiety about peer intimacy, $t(637)=-3.94, p<.001$, than boys. In contrast, boys reported more parental rejection, $t(637)=$ $2.81, p<.001$, and more avoidance of peer intimacy, $t(637)=2.32, p<.05$, than girls (see Table 1$).$

\section{Rejection Experiences, RS and Loneliness Correlations.}

Correlations between all measures are shown in Table 2, with correlations for boys above the diagonal and for girls below the diagonal. All conditions for mediation were met, with associations between parent rejection, peer victimisation, anxious and angry RS, and loneliness significant at $p<.01$ (Baron \& Kenny, 1986). In support of Hypothesis 1, boys and girls were lonelier when they reported greater parent rejection and peer victimisation. Also, in support of Hypothesis 2, boys and girls were lonelier when they reported more anxious RS and when they reported more angry RS. One correlation differed between boys and girls: the association of loneliness with avoidance of intimacy was weaker in boys than in girls, $p<.05$.

\section{Anxious RS as a mediator.}

The first set of hierarchical multiple regression models was used to test anxious RS as a mediator of the association between rejection experiences and loneliness (Hypothesis 3). Overall, the results were similar for boys and girls, so the total sample results are reported. Children reporting more rejection by their parents (path $a=0.24, p<.01$; see Figure 1) or victimisation by their peers (path $a=$ $0.31, p<.01)$ reported more anxious $\mathrm{RS}, R^{2}=.15, F(2$, $636)=54.22, p<.001$. Children who reported more parent rejection $(B=0.26, p<.01)$ and peer victimisation $(B=0.39, p<.01)$ were lonelier, $R^{2}=.27, F(2$, $636)=119.58, p<.01$. In addition, those reporting more anxious RS were lonelier (path $b=0.22, p<.01$ ). The bias-corrected bootstrap $95 \%$ confidence intervals for the indirect effects of parental rejection $(a b=0.05)$ and peer victimisation $(a b=0.07)$ on loneliness via anxious RS did not include zero (parental rejection: 0.03 to 0.08 ; peer victimisation: 0.04 to 0.10 ). However, for both parent rejection and peer victimisation, the $c$ ' paths remained significant, suggesting only partial mediation. Thus, parental rejection and peer victimisation had direct associations with loneliness, but also an indirect associations, due to the mediating role of anxious RS. Overall, this model accounted for $33 \%$ of the variance in loneliness, $F(3,635)=103.53, p<.01$.

\section{Angry RS as a mediator.}

In the second set of hierarchical multiple regression models testing angry RS as a mediator (Hypothesis 3), similar 


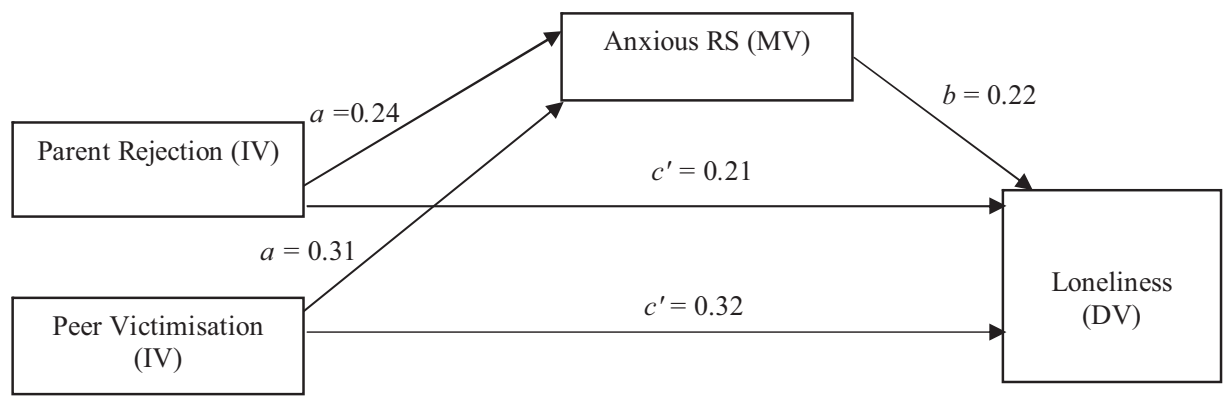

\section{FIGURE I}

The partial mediating effect $(N=639)$ of anxious $R S$ on the relationship between parent rejection, peer victimisation and loneliness. Note: Unstandardised regression coefficients (Bs) are shown.

analyses were conducted and the total sample results are reported. Children reporting more rejection by their parents (path $a=0.18, p<.01$; see Figure 2) or victimisation by their peers (path $a=0.30, p<.01$ ) also reported more angry RS, $R^{2}=.12, F(2,636)=41.74, p<.01$. In addition, those reporting more angry RS were lonelier (path $b=0.12, p<.05)$. The bias-corrected bootstrap 95\% confidence interval for the indirect effects of parental rejection $(a b=0.02)$ and peer victimisation $(a b=0.04)$ on loneliness via angry RS did not include zero (parental rejection: 0.01 to 0.04 ; peer victimisation: 0.02 to 0.06 ). In addition, for both parent rejection and peer victimisation the $c^{\prime}$ paths remained significant, although with a slight reduction, suggesting partial mediation. Thus, as was found for anxious RS, parental rejection and peer victimisation had direct associations with loneliness, but also an indirect associations, due to the mediating role of angry RS. Overall, this model accounted for $29 \%$ of the variance in loneliness, $F(3,635)=87.08, p<.01$.

\section{Loneliness, RS and Anxiety about Peer Intimacy}

Supporting Hypothesis 4, children who reported heightened RS had greater anxiety about peer intimacy and simple associations supported the possibility that children's anxiety about peer intimacy would mediate associations between RS and loneliness (Hypothesis 5; see Table 2). Therefore, two hierarchical regression models were estimated, one for anxious RS and one for angry RS, and results for the full sample are reported (results were similar for boys and girls). Children reporting more anxious RS (path $a=0.36, p<.01$; see Figure 3) or angry RS (path $a=0.31, p<.01$ ) also reported more anxiety about peer intimacy (anxious RS: $R^{2}=.16, F[1,637]=$ $116.90, p<.01$; angry RS: $R^{2}=.12, F[1,637]=84.30$, $p<.01)$. In addition, children reporting more anxiety about peer intimacy were lonelier (path $b=0.11, p<$ .05 in the anxious RS model; path $b=0.17, p<.01$ in the angry RS model). Children who reported more anxious RS $(B=0.36, p<.01)$ or angry $\mathrm{RS}(B=0.26$, $p<.01)$ were lonelier. The bias-corrected bootstrap $95 \%$ confidence interval for the indirect effect of anxious RS $(a b=0.04)$ and angry RS $(a b=0.05)$ on loneliness via anxiety about peer intimacy did not include zero (anxious RS: 0.01 to 0.07 ; angry RS: 0.03 to 0.08 ). Also, for both anxious and angry RS, the $c$ paths remained significant, although with a slight reduction. This indicated that anxious and angry RS have direct associations with loneliness, but they also had partial indirect associations due to the mediating role of anxiety about peer intimacy. Overall, the anxious RS model accounted for 19\% of the variance, $F(2,636)=72.36, p<.01$, and the angry $\mathrm{RS}$

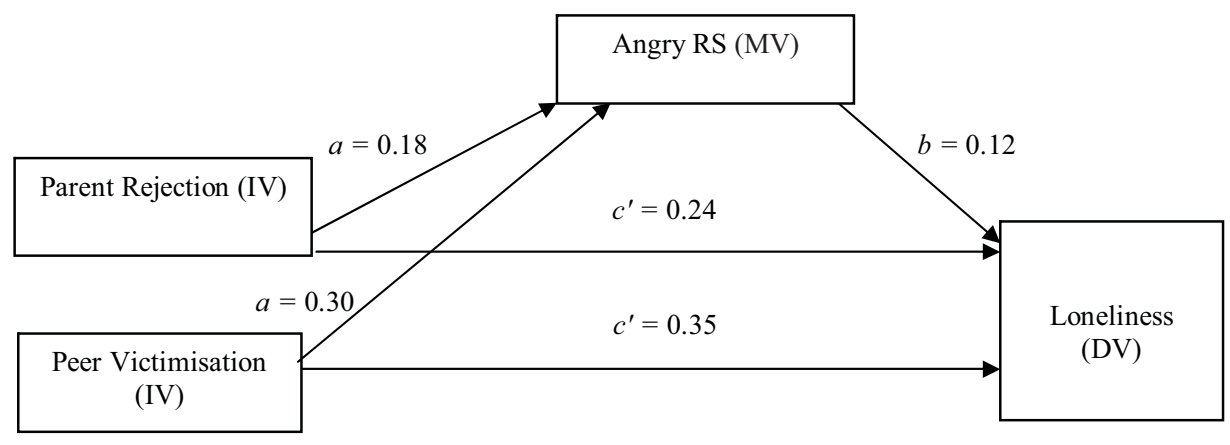

\section{FIGURE 2}

The partial mediating effect $(N=639)$ of angry RS on the relationship between parent rejection, peer victimisation and loneliness. Note: Unstandardised regression coefficients (Bs) are shown. 


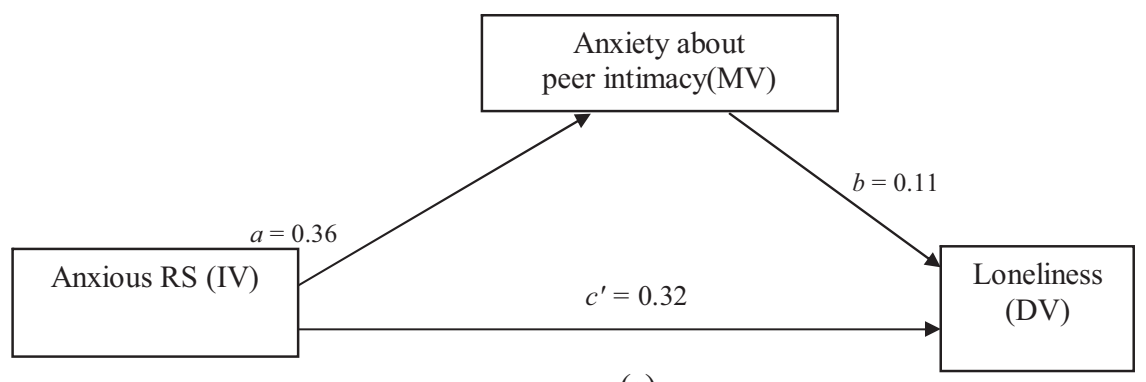

(a)

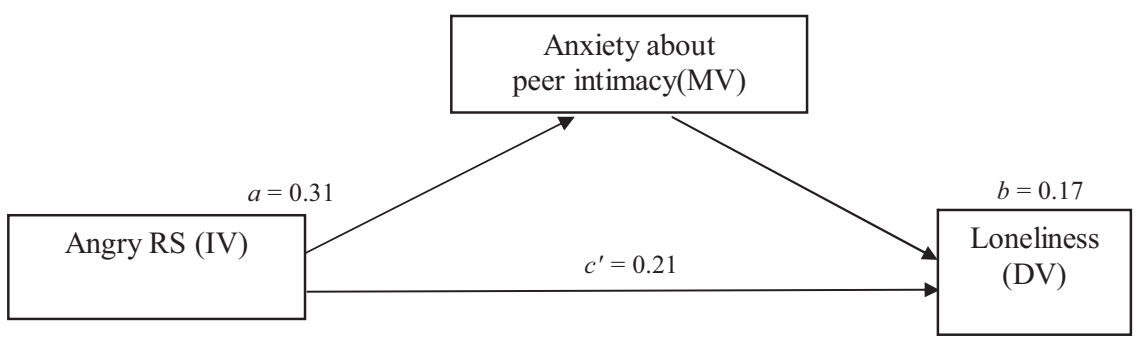

(b)

\section{FIGURE 3}

The partial mediating effect $(N=639)$ of anxiety about peer intimacy on the relationship between anxious RS and loneliness (Figure 3a) and between angry $\mathrm{RS}$ and loneliness (Figure $3 \mathrm{~b}$ ).

Note: Unstandardised regression coefficients (Bs) are shown.

model accounted for $12 \%$ of the variance in loneliness, $F(2,636)=44.20, p<.01$.

\section{Loneliness, RS and Avoidance of Peer Intimacy}

Supporting Hypothesis 6, boys and girls who reported greater rejection sensitivity were more likely to avoid peer intimacy (see Table 2) and simple associations supported the possibility that children's avoidance of about peer intimacy would mediate associations between RS and loneliness (Hypothesis 7; see Table 2). However, some differences were found for boys and girls, so results are reported for each group.

The association between boys' loneliness and RS, both anxious and angry, with the introduction of intimacy avoidance, were not significant, and bootstrapping results also indicated no mediating effect of avoidance of peer intimacy among boys. The results of two hierarchical regressions, one for anxious RS and one for angry RS, showed that girls who reported more anxious RS (path $a=0.24, p<.01$; see Figure 4) or angry RS (path $a=0.17, p<.01)$ reported more avoidance of peer intimacy (anxious RS: $R^{2}=.09, F[1,308]=28.54, p<$ .01 ; angry RS: $\left.R^{2}=.04, F[1,308]=13.13, p<.01\right)$. In addition, girls reporting more avoidance were lonelier (path $b=0.26, p<.01$ in the anxious RS model; path $b=0.31, p<.01$ in the angry RS model). The bias-corrected bootstrap $95 \%$ confidence interval for the indirect effect of anxious RS $(a b=0.06)$ and angry RS $(a b=0.05)$ on loneliness via avoidance of peer intimacy did not include zero (anxious RS: 0.03 to 0.11 ; angry RS: 0.02 to 0.09 ). For both anxious and angry RS, the $c^{\prime}$ paths remained significant, although with a slight reduction. This indicated that, for girls, anxious and angry RS have direct associations with loneliness, but there are also partial indirect associations due to the mediating role of avoidance. Overall, the anxious RS model accounted for $22 \%$ of the variance in loneliness, $F(2,307)=42.12$, $p<.01$ and the angry RS model accounted for $17 \%$ of the variance in loneliness, $F(2,307)=31.06, p<.01$.

\section{Discussion}

The purpose of this study was to test whether rejection by parents and victimisation by peers are social correlates of loneliness and to determine whether preadolescents' emotional sensitivities play a role in explaining links between rejection experiences and loneliness. In general, the present study's findings support theories that detail how rejection by parents and peers facilitates the development of emotional sensitivities to relationship problems (Cassidy \& Berlin, 1999). As tested here, it also seems to be the case that anxious and angry RS arise most directly from these rejection experiences, which in turn may exacerbate more specific sensitivities about peers 


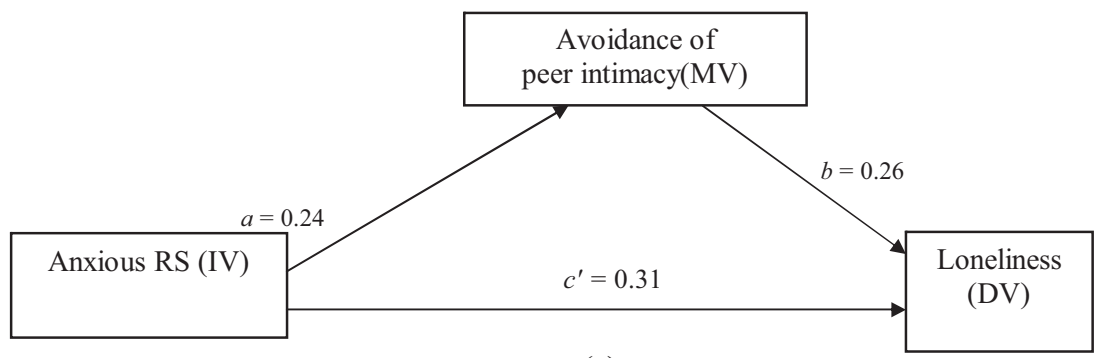

(a)

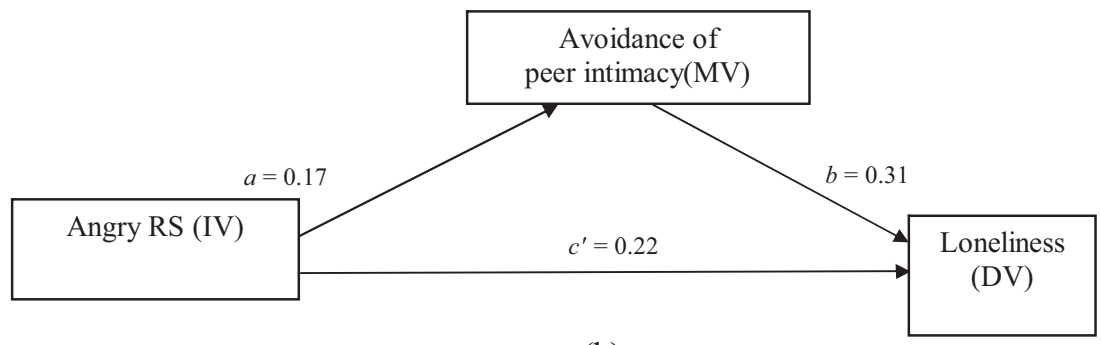

(b)

\section{FIGURE 4}

The partial mediating effect of girls' $(n=310)$ avoidance of peer intimacy on the relationship between anxious RS and loneliness (Figure 4a) and angry RS and loneliness (Figure 4b).

Note: Unstandardised regression coefficients (Bs) are shown.

relationships, measured here as anxiety about peer intimacy and avoidance of intimacy. The findings regarding the mediational roles of emotional sensitivities in linking rejection to loneliness also provide support for the view that these sensitivities increase the likelihood of further relationship problems, culminating in loneliness and maladjustment in preadolescence (Downey et al., 1999).

\section{Rejection Experiences, RS and Loneliness}

First, as anticipated and consistent with previous research (Downey \& Feldman, 1996; London et al., 2007; McDonald et al., 2010), heightened perceptions of parental rejection and victimisation by peers were each associated with greater RS, which in turn were associated with adolescents' greater feelings of loneliness. These findings extend those reported by London et al. (2007), by further testing and refining the RS model (Downey \& Feldman, 1996). Although previous research had tested the associations of peer relationships and RS with loneliness (London et al., 2007), this was the first study to extend the RS model to test these associations, while also testing the unique role of parental rejection and examining indirect associations of rejection with loneliness via both anxious and angry RS. Thus, to our knowledge, this is the first study to show direct, unique associations with loneliness of preadolescents' rejection by both parents and peers, while also showing that some of the total effect of rejection on loneliness is indirect via anxious and angry RS.

\section{Loneliness, RS, Anxiety About Intimacy and Intimacy Avoidance}

Second, we introduced additional emotional sensitivities to extend the RS model's (Downey et al., 1999) finding that two sensitivities more specific to peer relationship interaction - anxiety about peer intimacy and avoidance of peer intimacy - were positively related to anxious and angry RS. No previous study has examined anxiety about peer intimacy and avoidance of intimacy as interpersonal sensitivities that might be linked with RS and further account for adolescents' loneliness. Given that the internal consistency of the measures of anxiety about intimacy and avoidance of intimacy were lower than ideal, these findings need replication, preferably with improved measures. Nonetheless, present findings add to previous research by Downey and colleagues (1999) that supported mediational links between rejection experiences and avoidance and overinvestment (anxiety) relationship strategies via RS. As expected, rejection sensitive adolescents in the present study were more likely to report anxiety about peer intimacy, consistent with previous research (Downey et al., 1999). Also, for both boys and girls, anxiety about peer intimacy mediated the association between RS and loneliness. Rejection sensitive adolescents were also more likely to report avoidance of intimacy in peer relationships, in accordance with work by Downey and colleagues (1999). However, in the present study, only for rejection sensitive girls, was perceived loneliness higher for those reporting more intimacy avoidance in their peer relationships. This suggests that for girls with already 
heightened expectations of rejection, avoidance of intimacy may serve to further emphasise the discrepancy between ideal and actual social relationships, thus capturing the very essence of loneliness.

\section{Limitations, Future Research and Implications}

All of these findings should be considered along with some study limitations. First, self-report was used to measure all constructs; although, in the present study, the selection and development of measures was guided by theoretical considerations, which suggest that selfreport is one of the most reliable sources of information about internal emotional states, such as loneliness (Ladd, Kochenderfer, \& Coleman, 1996). A second limitation is the use of cross-sectional design to imply directional pathways and to study mediation effects. It is possible, for example, that RS facilitates rejection experiences or that the relationship between these variables is reciprocal in nature. However, our findings in such a large sample of preadolescents provide important preliminary evidence to support future longitudinal research.

The third limitation relates to the scale utilised in the present study to measure intimacy avoidance and anxiety. This was adapted from a well-known and well-validated adult measure, which focuses on romantic relationships (Brennan et al., 1998). However, the attenuated internal consistency of this scale suggests its limited applicability to participants in preadolescence. Further, the scale emphasises eluding intimacy as the main source of avoidant behaviour, potentially limiting generalisability to avoidance in boys. Future research could focus on developing a measure that is more specific to preadolescence and one that incorporates alternate interpersonal relationship qualities (i.e., peer group and dyadic) that contribute to boys' avoidant strategies, which is a neglected area of research (Rose \& Rudolph, 2006). Despite these limitations, the present study provides preliminary evidence of associations between RS, loneliness and both avoidance and anxiety in preadolescence. This highlights the importance of continuing to study relationship behaviour patterns in rejection sensitive preadolescents in order to inform intervention design. At an individual level, it may be helpful to intervene early, at school, to identify lonely or rejection sensitive children. It may also be helpful to identify young people high in rejection sensitivity seeking services in order to address their emotional reactions to, and cognitive beliefs about, rejection, at the same time as promoting better social skills and providing opportunities for positive social experiences with peers and others. For example, previous research has shown that practical school curriculum design focused on promoting emotional wellbeing can reduce children's experiences of loneliness (Liepins \& Cline, 2011). Such an approach might be extended to focus more specifically on helping children to regulate their emotions and use more positive coping responses when they experience rejection or feel they are excluded or ostracised by others.

Future research could add to these findings by incorporating multiple contributory constructs of peer experiences (i.e., peer acceptance, friendship quality), to understand how they function synergistically to affect boys and girls over time. Previous research has suggested that in order to improve psychological adjustment, intervention work should target children's friendship experiences, with a focus on teaching gender and age-specific goals such as intimacy development and increasing the size of one's friendship network (Asher, Parker, \& Walker, 1996; Parker \& Asher 1993).

\section{Conclusion}

In sum, the present study provides further empirical support for the RS model (Downey et al., 1999) and expands on RS theory with the first evidence that the relationship behaviours avoidance and anxiety, depicted in the RS model, are applicable to preadolescence. Findings also suggest that loneliness in preadolescence is a social correlate of RS and further implies that gender differences are present in the mechanisms through which rejection sensitive biases are expressed. In particular, girls express more anxious RS and anxiety about peer intimacy, whereas boys express more avoidance of peer intimacy. Further, the associations between RS and loneliness was mediated via avoidance of intimacy for girls but not boys. This supports the expected centrality of intimate relationships for girls as opposed to the more agentic goals pursued by boys (Rose \& Rudolph, 2006). Future studies should continue to investigate the social antecedents and consequences of loneliness and RS in preadolescence.

\section{Acknowledgments}

This study was supported by a grant from the Australian Research Council DP1096183. We thank Professors Drew Nesdale and Geraldine Downey for their early input on this project. We also thank Leanne McGregor, Belinda Goodwin, Shawna Mastro and a large number of staff and volunteers who managed and assisted with data collection. Finally, we appreciate the approval for this project from Education Queensland and the school and student participation; without it, this project would not have been possible.

\section{References}

Asher, S.R., \& Paquette, J.A. (2003). Loneliness and peer relations in childhood. Current Directions in Psychology, 12, 75-78. doi:org/10.1097/00011363-199902000-00004

Asher, S.R., Parker, J.G., \& Walker, D.L. (1996). Distinguishing friendship from acceptance: Implications for intervention and assessment. In W.M. Bukowski, A.F. Newcomb, \& W.W. Hartup (Eds.), The company they keep: Friendship during 
childhood and adolescence (pp. 366-405). New York: Cambridge University Press.

Baron, R.M., \& Kenny, D.A. (1986). The moderator-mediator variable distinction in social psychological research: Conceptual, strategic, and statistical considerations. Journal of Personality and Social Psychology, 51, 1173-1182.

Berndt, T.J., \& Ladd, G. (1989). Peer relationships in child development. New York: Wiley.

Bowlby, J. (1969). Attachment and loss (vol. 1). New York: Basic Books.

Brennan, K.A., Clark, C.L., \& Shaver, PR. (1998). Self-report measurement of adult attachment: An integrative overview. In J.A. Simpson \& W.S. Rholes (Eds.), Attachment theory and close relationships (pp. 46-76). New York: Guilford.

Cassidy, J., \& Asher, S.R. (1992). Loneliness and peer relations in young children. Child Development, 63, 350-365. doi:org/10.1111/j.1467-8624.1992.tb01632

Cassidy, J., \& Berlin, L.J. (1999). Understand the origins of childhood loneliness: Contributions of attachment theory. In K.J. Rotenberg \& S. Hymel (Eds.), Loneliness in childhood and adolescence (pp. 34-55). New York: Cambridge University Press.

Crick, N.R., \& Grotpeter, J.K. (1996). Children's treatment by peers: Victims of relational and overt aggression. Development and Psychopathology, 8, 367-380. doi:org/10.1017/S0954579400007148

Downey, G., Bonica, G., \& Rincón, C. (1999). Rejection sensitivity and adolescent romantic relationships. In W. Furman, B. Bradford Brown, \& C. Feiring (Eds.), The development of romantic relationships in adolescence (pp. 148-174). New York: Cambridge University Press.

Downey, G., \& Feldman, S.I. (1996). Implications of rejection sensitivity for intimate relationships. Journal of Personality and Social Psychology, 70, 1327-1343. doi:org/10.1037//00223514.70.6.1327

Downey, G., Feldman, S., \& Ayduk, O. (2000). Rejection sensitivity and male violence in romantic relationships. Personal Relationships, 7, 45-61. doi:org/10.1111/j.14756811.2000.tb00003

Downey, G., Lebolt, A., Rincón, C., \& Freitas, A. L. (1998). Rejection sensitivity and children's interpersonal difficulties. Child Development, 69, 1074-1091. doi:org/10.1111/j.14678624.1998.tb06161.x

Duarte, L.M., \& Thompson, L.M. (1999). Sex differences in self-silencing. Psychological Reports, 85, 145-161. doi:org/10.2466/pr0.1999.85.1.145

Gilligan, C. (1982). In a different voice. Cambridge: Harvard University Press.

Harper, M.S., Dickson, J.W., \& Welsh, D.P. (2006). Selfsilencing and rejection sensitivity in adolescent romantic relationships. Journal of Youth and Adolescence, 124, 5-17. doi:org/10.1007/s10964-006-9048-3

Hawker, D.S., \& Boulton, M.J. (2000). Twenty years' research on peer victimisation and psychosocial maladjustment: A metaanalytic review of cross-sectional studies. Journal of Child Psychology and Psychiatry, 4, 441-455. doi:10.1111/14697610.00629

Hayes, A.F. (2012). PROCESS: A versatile computational tool for observed variable mediation, moderation, and conditional process modeling [White paper]. Retrieved from http://www. afhayes.com/ public/process2012.pdf
Heinrich, L.M., \& Gullone, E. (2006). The clinical significance of loneliness: A literature review. Clinical Psychology Review, 26, 695-718. doi:org/10.1016/j.cpr.2006.04.002

Johnson, H.D., LaVoie, J.C., Spenceri, M.C., \& Mahoney-Wernli, M.A. (2001). Peer conflict avoidance: Associations with loneliness, social anxiety, and social avoidance. Psychological Reports, 88, 227-235. doi: org/10.2466/pr0.2001.88.1.227

Kingery, J.N., Erdley, C.A., \& Marshall, K.C. (2011). Peer acceptance and friendship as predictors of early adolescents' adjustment across the middle school transition. Merrill-Palmer Quarterly, 57, 215-243. doi:org/10.1353/mpq.2011.0012

Ladd, G.W., Kochenderfer, B.J., \& Coleman, C.C. (1996). Friendship quality as a predictor of young children's early school adjustment. Child Development, 67, 1103-1118. doi:org/10.2307/1131882

Laursen, B., \& Hartl, A.C. (2013). Understanding loneliness during adolescence: Developmental changes that increase the risk of perceived social isolation. Journal of Adolescence, 36, 12611268. doi:org/10.1016/j.adolescence.2013.06.003

Liepins, M., \& Cline, T. (2011). The development of concepts of loneliness during the early years in school. School Psychology International, 32, 397-411. doi:org/10.1177/0143034311404132

London, B., Downey, G., Bonica, C., \& Paltin, I. (2007). Social causes and consequences of rejection sensitivity. Journal of Research on Adolescence, 17, 481-506. doi:org/10.1111/j.15327795.2007.00531.x

Ludlow, L.H., \& Mahalik, J.R. (2001). Congruence between a theoretical continuum of masculinity and the rasch model: Examining the conformity to masculine norms inventory. Journal of Applied Measurement, 2, 205-226.

Marston, E.G., Hare, A., \& Allen, J.P. (2010). Rejection sensitivity in late adolescence: Social and emotional sequelae. Journal of Research on Adolescence, 20, 959-982. doi:org/10.1111/j.15327795.2010.00675

McDonald, K.L., Bowker, J.C., Rubin, K.H., Laursen, B., \& Duchene, M.S. (2010). Interactions between rejection sensitivity and supportive relationships in the prediction of adolescents' internalizing difficulties. Journal of Youth Adolescence, 39, 563-574. doi:org/10.1007/s10964-010-9519-4

Orpinas, P., \& Horne, A. (2006). Bullying prevention: Creating a positive school climate and developing social competence interactions. Washington, DC: American Psychological Association.

Ozen, A., Sumer, N., \& Demir, M. (2010). Predicting friendship quality with rejection sensitive attachment security. Journal of Social and Personal Relationships, 28, 163-181. doi:org/10.1177/0265407510380607

Parker, J.G., \& Asher, S.R. (1993). Friendship and friendship quality in middle childhood: Links with peer group acceptance and feelings of loneliness and social dissatisfaction. Developmental Psychology, 29, 611-621. doi:org/10.1037/00121649.29.4.611

Preacher, K.J., Rucker, D.D., \& Hayes, A.F. (2007). Assessing moderated mediation hypotheses: Theory, methods, and prescriptions. Multivariate Behavioral Research, 42, 185-227.

Purdie, V., \& Downey, G. (2000). Rejection sensitivity and adolescent girls' vulnerability to relationshipcentered difficulties. Child Maltreatment, 5, 338-349. doi:org/10.1177/1077559500005004005

Rose, A.J., \& Rudolph, K.D. (2006). A review of sex differences in peer relationship processes: Potential trade-offs for 
the emotional and behavioural development of boys and girls. Psychological Bulletin, 132, 98-131. doi:org/10.1037/00332909.132.1.98

Rosenfeld, L.B. (1979). Self-disclosure avoidance: Why am I afraid to tell you who I am? Communication Monographs, 46, 63-74. doi:org/10.1080/03637757909375991

Rubin, K.H., Bukowski, W., \& Parker, J.G. (1998). Peer interactions, relationships, and groups. In W. Damon \& N. Eisenberg (Eds.), Handbook of child psychology (5th ed.): Vol 3: Social, emotional, and personality development (pp. 619-700). New York: Wiley. doi:10.1002/9780470147658.chpsy0310

Rubin, K.H., \& Coplan, R.J. (2004). Paying attention to and not neglecting social withdrawal and social isolation. Merrill-Palmer Quarterly, 50, 506-534. doi:org/10.1353/mpq.2004.0036

Sentse, M., Lindenberg, S., Omvlee, A., Ormel, J., \& Veemstra, R. (2010). Rejection and acceptance contexts: Parents and peers as risks and buffers for early adolescent psychopathology. The
TRAILS study. Journal of Abnormal Child Psychology, 38, 119130. doi:org/10.1007/s10802-009-9351-z

Skinner, E.A., Johnson, S., \& Snyder, T. (2005). Six dimensions of parenting: A motivational model. Science and Practice, 5, 175-235. doi:org/10.1207/s15327922par0502_3

Ward, C.A., Bergner, R.M., \& Kahn, J.H. (2003). Why do men distance? Factors predictive of male avoidance of intimate conflict. Family Therapy, 30, 1-11.

Zimmer-Gembeck, M.J., Geiger, T.C., \& Crick, N.R. (2005). Relational and physical aggression, prosocial behaviour and peer relations: Gender moderation and bidirectional Associations. The Journal of Early Adolescence, 25, 421-452. doi:org/10.1177/0272431605279841

Zimmer-Gembeck, M.J., \& Nesdale, D. (2013). Anxious and angry rejection sensitivity, social withdrawal and retribution in high and low ambiguous situations. Journal of Personality, 81, 29-38. doi:10.1111/j.1467-6494.2012.00792 